\title{
Community Health Officer Post - Potential Concerns
}

\section{Vishalkumar J Jani}

Assistant Professor, Indian Institute of Public Health Gandhinagar, Gandhinagar, Gujarat 382042

\begin{tabular}{|l|l|l|l|l|l|l|l|}
\hline Abstract & Introduction & Methodology & Results & Conclusion & References & Citation & Tables/ Figures \\
\hline
\end{tabular}

\section{Corresponding Author}

Dr. Vishalkumar J. Jani, Assistant Professor, Indian Institute of Public Health Gandhinagar, Gandhinagar, Gujarat 382042

E Mail ID: vjani@iiphg.org

\section{Citation}

Jani VJ. Community Health Officer Post - Potential Concerns. Indian J Comm Health. 2020;32(4):743-745. https://doi.org/10.47203/IJCH.2020.v32i04.024

Source of Funding: Nil Conflict of Interest: None declared

\section{Article Cycle}

Received: 07/11/2020; Revision: 28/11/2020; Accepted: 30/12/2020; Published: 31/12/2020

This work is licensed under a Creative Commons Attribution 4.0 International License.

\section{Abstract}

Under Ayushman Bharat Health and Wellness Centre (HWC) initiative, a middle-level health provider post, named Community Health Officer ( $\mathrm{CHO}$ ), is envisioned to bridge the gap between the health system and community. This cadre has multiple roles and responsibilities that mirror what used to be done by the Auxiliary Nurse Midwife (ANM) at sub-center before conversion to HWC. Owing to educational and experience requirements of $\mathrm{CHO}$, and existing other cadres at the sub-center, there may be some concerns related to role ambiguity, interpersonal issues, inter- and intra-cadre conflicts, and non-cooperation challenges.

\section{Keywords}

Ayushman Bharat; Community Health Officer

\section{Introduction}

India, with an ambition of achieving universal health coverage (UHC), launched Ayushman Bharat scheme in 2018. The scheme has two parts: (i) Pradhan Mantri Jan Arogya Yojana (PMJAY) that intends to provide financial protection to the population against unforeseen health expenditure; (ii) Health and Wellness Centres that intends to extend comprehensive primary healthcare by upgrading existing health centers, including primary health centers (PHC) and sub-health centers. As of Nov. 2020, Ayushman Bharat HWC (1) portal shows that there are 49419 operational HWCs in India. Out of these, 27719 have been converted from Sub-centres, 18094 from rural PHCs, and 3606 from urban PHCs (UPHC). The goal is to convert 1.5 lac sub-centers to HWCs by 2022.

The Health and Wellness Center conversion of subhealth centers involves an introduction of a new layer of health workers called Community Health
Officers $(\mathrm{CHO})$ in the Indian public health system. Except for this, there is no direct recruitment on any post under HWC converted facilities. The roles and responsibilities of the $\mathrm{CHO}$ reflect those of the midlevel healthcare provider (MLHP). CHOs can have a background in nursing (BSc or GNM), community health (BSC), or AYUSH.(2)

The introduction of new MLHP is mainly to expand the services available at the base level of the Indian public health system - sub-centers. The evident difference would be starting a quasi-OPD (outdoor patient department) at the sub-center HWC. The $\mathrm{CHO}$ may start diagnosing and dispensing some of the basic medicines at his/her level. With $\mathrm{CHO}$ available at HWC, many patients with NCDs who require long-term medications may visit sub-centre HWC and get medications, provided they have a prescription from the medical officer (MO), PHC. This results in more compliance as patients need not travel to PHC just to get medicines. For, some of the 
walk-in patients, $\mathrm{CHOs}$ are entitled to used telemedicine and contacting $\mathrm{MO}$ at PHC through the technology and directly dispense medicines to the patients. This also reduces patient load at PHCs, and gatekeeping is in place.

$\mathrm{CHO}$ is a new cadre in the system and it is expected to evolve over the years. They need to play the roles of a provider of healthcare services, a manager, a leader, a supervisor, and an active participant in community healthcare.(2) The community at large is going to benefit with expanded scope of services at sub-center level HWCs, and the presence of a $\mathrm{CHO}$ who can be a very good connector between community and MO. However, along with the creation of a new cadre, some of the administrative and managerial issues are bound to occur. It is imperative to draw the attention of policymakers to such issues.

\section{Possible Issues with $\mathrm{CHO}$ post}

The way $\mathrm{CHO}$ post has been envisioned and operationalized there are few issues of parity across cadres, and within cadre that may arise. The relationship of $\mathrm{CHO}$ at each sub-center HWC with ANM or Staff-nurse is a potential area of concern. Each sub-center (before HWC conversion) had an ANM who was responsible for the operations of the center and coordination with the PHC. Essentially, ANM was the face of the sub-center and was part of all village level committees related to healthcare and sanitation. She was well-known to the community and the village administrators. Now, with the $\mathrm{CHO}$ cadre in place, the ANM would be supervised even at the sub-center level. And, ultimate responsibility of the operations, administration, data integrity, and coordination with the upper level lies with the $\mathrm{CHO}$. This may not directly result in the conflict between the ANM and the $\mathrm{CHO}$, but may cause a bit of cooperation issues as ANM may feel demotivated and demoted within the system. Unwanted behavioral issues may be a result of role ambiguity, especially from the ANM's perspective.

The above problem gets intense when most of the CHOs would have less work experience compared to that of an ANM who would most likely be a regular government employee for the last few years. This may have serious implications. The ANM may not really cooperate and share the understanding of the health needs and work of HWC with the $\mathrm{CHO}$. S/he may not have positive motivation with someone less experienced and very new to the field supervising his/her work. For the $\mathrm{CHO}$, there may be a direct comparison with the ANM in terms of payment and job security. These two factors and non-supportive behavior of ANM may result in major deterrents for the engagement of $\mathrm{CHO}$ in the work. The issue of experience may be taken care of if the $\mathrm{CHO}$ is from AYUSH background.

Another issue with the ANM - $\mathrm{CHO}$ relationship is the power ANM used to enjoy because of her involvement with the village administration, and $\mathrm{MO}$ of the primary health center. The system may introduce a new cadre between ANM and MO, but the ability of connection and familiarity of ANM with MO may cause many communication issues leading to the conflict. The MO has to be a moderator of this situation and resolve any issues amicably, and take care of this relation proactively. Assessing the impact of the creation of a new cadre called $\mathrm{CHO}$, with its operational guidelines as of today, would require extensive studies of behavioral changes in ANM, relationship dynamics of ANM-CHO, impact of ANM on the $\mathrm{CHO}-\mathrm{MO}$ relationship.

Apart from this CHO- ANM relationship, within the $\mathrm{CHO}$ cadre, there would be the presence of professionals from different backgrounds. Given the scenario in India, an AYUSH qualified person would be able to leverage more from the community as s/he would be seen as a doctor. S/he would be technically more knowledgeable compared to a nursing graduate taking up the $\mathrm{CHO}$ position. It may happen that HWCs manned by AYUSH CHOs would have more OPD footfalls compared to the other HWCs. There is a possibility that the $\mathrm{MO}-\mathrm{CHO}$ relationship would also be impacted due to the educational qualification. For testing this conjecture, future studies around outcomes like OPD footfalls, medicines, and other consumables consumption at HWCs may be required.

\section{Potential Changes for the $\mathrm{CHO}$ post}

The $\mathrm{CHO}$ cadre is going to be pivotal in expanding the comprehensive primary health care coverage. However, based on the above discussion, it is apparent while designing the cadre some of the issues had to be considered. One of the main issues is the relationship between ANM and $\mathrm{CHO}$. This would have been tackled by extending an option to the existing ANMs to become CHOs. The system would have recruited contractual employees at a lower level to cater to expanded scope of services. This would have resulted in dual advantage: 
avoidance of issues raised above and MLHPs would

have had a substantial field experience before taking up the job. The other option was to keep an entry requirement of substantial public health experience to become a $\mathrm{CHO}$. This would have resulted in less dissonance in the ANM cadre as they would not have to report or coordinate with someone who is less experienced.

As of now, there needs to be training and additional push to the $\mathrm{MO}$ to look after the $\mathrm{CHO}$ and facilitate their integration into the system. The MOs may be made aware of these probable issues and would be asked to play a proactive moderating role so that some of the conflicts can be avoided. This is for the larger benefits of the system and already converted HWCs.

\section{Conclusion}

Any change in the system would bring certain challenges even though the change is for the benefit of the overall community and system. The system is dependent on the persons it employs and their behavioral issues may have an impact on how the system functions. $\mathrm{CHO}$ is one of these changes that has been brought to expand the scope of services of sub-center but its efficiency and effectiveness depend on the various interrelationships amongst the different employees of the system. The policymakers and state administration need to take note of this and proactively design an intervention to handle these issues. The issue on its own seems to be small but with every potential sub-center HWC, this gets multiplied in inefficiency in the overall system.

\section{References}

1. Ayushman Bharat HWC portal [homepage on the Internet]. New Delhi: HWC related data. Available from https://abhwc.nhp.gov.in/ (Last Accessed on 2020 Dec 20)

2. National Health System Resource Centre, Ayushman Bharat: Comprehensive Primary Health Care through Health and Wellness Centres - Operational Guidelines, Available from https://ab-hwc.nhp.gov.in/ (Last Accessed on 2020 Dec 20) 